Journal of

Epilepsy and

Clinical

Neurophysiology

J Epilepsy Clin Neurophysiol 2008; 14(3):129-133

\title{
Considerações e Recomendações para o Uso de Formulações de Drogas Antiepilépticas
}

\section{Considerations and recommendations for use of generics antiepileptic drugs}

\author{
Carlos A. M. Guerreiro, Carlos E. Silvado, Elza M. Yacubian, Fernando Cendes, \\ Jaderson C. da Costa, Wagner Teixeira \\ Comissão de Genéricos de Drogas Antiepilépticas da Liga Brasileira de Epilepsia
}

\section{INTRODUÇÃO}

O tema sobre o uso de medicações genéricas tem sido bastante atual e de extrema relevância para a prática médica. Assim, a Liga Brasileira de Epilepsia (LBE), através de seu presidente, nomeou uma comissão de especialistas para debater o tema e apresentá-lo para discussão no Congresso Brasileiro de Epilepsia, em 13 de junho de 2008, estabelecendo a posição oficial da LBE sobre o tema. O tema amplo, polêmico, sem dados definitivos pela falta de estudos adequados do ponto de vista metodológico, foi discutido pela comissão e pela audiência.

\section{CONCEITOS COM REGULAMENTAÇÃO DA ANVISA}

Os medicamentos podem ser classificados em quatro categorias: 1. medicamentos inovadores, de referência ou originais; 2 . medicamentos licenciados; 3. medicamentos similares ou "cópias" e 4. medicamentos genéricos.

1 Medicamentos inovadores, de referência ou originais

Contém a entidade química (fármaco) desenvolvida e investigada originalmente pelo laboratório fabricante. $\mathrm{O}$ fabricante é o responsável pelos ensaios pré-clínicos e clínicos, nos quais foram demonstradas a eficácia e a segurança para as indicações clínicas e as condições de uso autorizadas. Geralmente o medicamento inovador é considerado medicamento de referência. Neste texto será referida como medicamento de referência.

\section{Medicamentos licenciados}

Especialidade igual ao medicamento de referência, comercializada por outra companhia com autorização do investigador; que fornece todas as informações assumindo, assim, a responsabilidade pelo medicamento em todos os aspectos de qualidade, segurança e eficácia.

\section{Medicamentos similares ou "cópias"}

Especialidade que contém o mesmo ou os mesmos princípios ativos do medicamento de referência, com a mesma concentração, forma farmacêutica, via de administração, posologia e indicação terapêutica, sendo equivalente ao medicamento registrado no órgão federal responsável pela vigilância sanitária. Pode diferir somente em características relativas ao tamanho e forma do produto, prazo de validade, embalagem, rotulagem, excipientes e veículos. Também chamados medicamentos me-too drugs (ou drogas "eu também"). Para os medicamentos similares é obrigatória a utilização de um nome comercial ou marca (nome fantasia), sendo proibida a utilização da denominação genérica. No Brasil, a Agência Nacional de Vigilância Sanitária (Anvisa) determinou que, para renovar os seus registros, os medicamentos similares deverão demonstrar que são equivalentes farmacêuticos ao medicamento de referência, ou seja, apresentam-se sob a mesma forma farmacêutica, contém idêntica composição qualitativa e quantitativa de princípio(s) ativo(s), e tem biodisponibilidade comparável, quando administrados na mesma dose molar e nas mesmas condições experimentais.

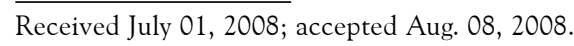




\section{Especialidade farmacêutica genérica}

Medicamento equivalente a um produto de referência ou inovador, que pretende ser com este intercambiável, geralmente é produzido após a expiração ou renúncia da proteção patentária ou de outros direitos de exclusividade, comprovada a sua eficácia, segurança e qualidade. É designado pela DCB (Denominação Comum Brasileira) ou, na sua ausência, pela DCI (Denominação Comum Internacional). No Brasil, o medicamento, para ser considerado genérico, deve demonstrar ser bioequivalente ao produto de referência e, por lei, podem ser intercambiáveis, isto é, substituir nas mesmas doses e apresentações os medicamentos de referência.

A Tabela 1 resume as características das formulações dos medicamentos no Brasil.

Tabela 1. Resumo das características dos medicamentos no Brasil.

\begin{tabular}{ll}
\hline $\begin{array}{c}\text { Medicamentos de referência } \\
\text { ou inovadores }\end{array}$ & \multicolumn{1}{c}{$\begin{array}{c}\text { Marca; patente; } \\
\text { estudos clínicos }\end{array}$} \\
\hline Medicamentos genéricos & $\begin{array}{l}\text { Denominação comum; } \\
\text { equivalência farmacêutica e } \\
\text { bioequivalência; intercambiáveis } \\
\text { por lei }\end{array}$ \\
\hline Medicamentos similares & $\begin{array}{l}\text { Nome fantasia; equivalência } \\
\text { farmacêutica e bioequivalência } \\
\text { (RDC 134/2003); pela lei, não } \\
\text { são considerados } \\
\text { intercambiáveis com a } \\
\text { referência }\end{array}$ \\
\hline
\end{tabular}

\section{Teste de Bioequivalência}

O teste de bioequivalência deve ser realizado com um número mínimo de 12 voluntários sadios. Estes são préselecionados por avaliações físicas e exames clínico-laboratoriais e são internados em duas ocasiões. Na primeira, recebem uma dose única do medicamento de referência ou do medicamento candidato a similar ou genérico e, depois de transcorrido o tempo necessário para que o organismo elimine $90 \%$ do fármaco, os voluntários são internados novamente e aqueles que receberam o medicamento de referência receberão o medicamento candidato a similar ou genérico e vice-versa. Esses voluntários são internados na noite anterior à realização do estudo, após a ingestão de lanche padronizado, o que garante a permanência em jejum por cerca de 10 horas. Antes da administração dos medicamentos, é coletada uma amostra sangüínea de cada voluntário, que servirá de controle para a certificação de que não há fármaco no organismo. Após a administração do medicamento, são coletadas amostras sangüíneas, conforme um cronograma pré-estabelecido, no qual se garante a absorção de mais de $80 \%$ do fármaco. As amostras serão utilizadas para quantificar o fármaco absorvido. Os valores de concentração de fármaco são plotados em gráfi- co, obtendo-se uma curva de concentração plasmática em função do tempo. A partir dessa curva, são calculados os parâmetros farmacocinéticos necessários para estabelecer a bioequivalência: a área sob a curva (ASC), pico de concentração (Cmax) e tempo do pico máximo (Tmax). Tais parâmetros são comparados estatisticamente. $\mathrm{O}$ teste de bioequivalência envolve três etapas: clínica, analítica e estatística. Seja T (de teste) o medicamento candidato a similar ou genérico e R o de referência. Esses dois medicamentos serão considerados bioequivalentes quando as razões dos parâmetros farmacocinéticos ASC (T)/ASC (R) e Cmáx (T)/Cmáx (R) estiverem contidos no intervalo de confiança de $90 \%$ (IC90\%), dentro do limite de $80 \%$ a $125 \%$.

Esta exigência está regulamentada pela Resolução da Diretoria Colegiada da Anvisa (Resolução-RDC) no 133/ 2003. Para um melhor entendimento, o registro dos medicamentos na ANVISA é revalidado a cada cinco anos. $\mathrm{Na}$ ocasião da primeira revalidação, após 1 - de dezembro de 2004, por exemplo, para medicamentos de prescrição médica, a empresa detentora do registro deverá apresentar o estudo de equivalência farmacêutica e bioequivalência. Caso não tenha realizado ainda o teste de bioequivalência, deverá obrigatoriamente apresentá-lo na ocasião da segunda revalidação, a partir de 1 de dezembro de 2009. Os medicamentos registrados ou revalidados antes de junho de 2003, data da publicação da legislação de similares, deverão ser revalidados até 02 de junho de 2008. Como o estudo de bioequivalência poderá ser apresentado até a segunda revalidação, todos os medicamentos similares deverão estar regulamentados até 2 de junho de 2013, ou seja, a partir desta data, todos os produtos similares obrigatoriamente terão apresentado os estudos de equivalência farmacêutica e bioequivalência. A Resolução-RDC no 134/2003 estabelece que esta demonstração ocorrerá de acordo com criticidade dos princípios ativos e classes terapêuticas e também com a data de renovação do registro do medicamento. Para os medicamentos mais críticos (entre eles drogas antiepilépticas com faixa terapêutica estreita, pouca solubilidade em água e cinética não linear) a data limite foi estabelecida para 01/12/2004. Por este motivo diversos medicamentos que não apresentaram os respectivos estudos de bioequivalência tiveram seus registros cancelados, conforme nota divulgada no site da ANVISA (Tabela 2) Estes antiepilépticos são considerados medicamentos similares de dose crítica, e apesar de terem comprovado a equivalência farmacêutica e bioequivalência até 01/12/2004, não são considerados intercambiáveis com os medicamentos de referência, não podendo ser substituídos ou substituir outros medicamentos de referencia e genéricos. Diversos outros antiepilépticos comercializados no Brasil não foram incluídos na lista de medicamentos similares de dose crítica e, portanto, não estão obrigados a apresentar estudos de bioequivalência antes de 02 de junho de 2013. 
Tabela 2. Antiepilépticos considerados medicamentos de dose crítica pela ANVISA e seus respectivos medicamentos de referência.

\begin{tabular}{lc}
\hline Medicamentos de dose crítica & $\begin{array}{c}\text { Medicamento de referência / } \\
\text { Fabricante }\end{array}$ \\
\hline $\begin{array}{l}\text { Ácido valpróico } \\
\text { Cápsulas }\end{array}$ & Depakene / Abbott \\
\hline $\begin{array}{l}\text { Carbamazepina } \\
\text { Comprimidos e suspensão oral }\end{array}$ & Tegretol / Novartis \\
\hline $\begin{array}{l}\text { Fenitoina } \\
\text { Comprimidos, Cápsulas e } \\
\text { suspensão oral }\end{array}$ & Hidantal / Aventis \\
\hline $\begin{array}{l}\text { Oxcarbazepina } \\
\text { Comprimidos e suspensão oral }\end{array}$ & Trileptal / Novartis \\
\hline $\begin{array}{l}\text { Primidona } \\
\text { Comprimidos e suspensão oral }\end{array}$ & Epidona / Wyeth Whitehall \\
\hline
\end{tabular}

\section{A prescrição de medicamentos genéricos no Brasil}

A regulamentação do uso de genéricos no Brasil é definida pela Lei n⿳9 9.787, de 10 de fevereiro de 1999, que altera a Lei $\mathrm{n}^{\mathrm{o}}$ 6.360, de 23 de setembro de 1976, dispõe sobre a vigilância sanitária, estabelece o medicamento genérico, dispõe sobre a utilização de nomes genéricos em produtos farmacêuticos e dá outras providências e diversos Regulamentos Técnicos de Medicamentos Genéricos (RDC). Informações mais detalhadas sobre a legislação e genericos no Brasil poderão ser encontradas em < http://www.anvisa.gov.br/hotsite/genericos/index. htm $>{ }^{1}$ e $<$ http://e-legis.bvs.br/leisref/public/home.php $>.^{2}$ O RDC 135, de 29/05/03 determina como deve ser a prescrição de medicamentos genéricos no Brasil. No âmbito do Sistema Único de Saúde (SUS), as prescrições pelo profissional responsável adotarão, obrigatoriamente, a De nominação Comum Brasileira (DCB), ou, na sua falta, a Denominação Comum Internacional (DCI). Nos serviços privados de saúde, a prescrição ficará a critério do profissional responsável, podendo ser realizada sob nome genérico ou comercial. No caso de o profissional prescritor decidir pela não-intercambialidade de sua prescrição, a manifestação deverá ser efetuada por item prescrito, de forma clara, legível e inequívoca, devendo ser feita de próprio punho, não sendo permitidas outras formas de impressão.

\section{A prescrição de medicamentos similares}

Estes medicamentos só podem ser vendidos quando prescritos pelo médico com o seu nome de marca. $\mathrm{O}$ paciente pode solicitar ao farmacêutico a troca do medicamento de referência pelo genérico, mas não pelo similar. A troca pelo similar, deverá ter autorização do médico.

A Lista de medicamentos genéricos registrados sujeitos a controle especial (47ª edição-17/3/08), incluindo os antiepilépticos está disponível em <www.anvisa.gov.br/ divulga/informes/2008/250308_2.htm $>.^{3}$
A dispensação/intercambialidade entre os medicamentos - Regulamento técnico para medicamentos genéricos (RDC 135 - 29/05/03)

Será permitida ao profissional farmacêutico a substituição do medicamento prescrito pelo medicamento genérico correspondente, salvo restrições expressas pelo profissional prescritor. Nesses casos, o profissional farmacêutico deverá indicar a substituição realizada na prescrição, apor seu carimbo com o seu nome e número de inscrição do Conselho Regional de Farmácia, datar e assinar. Nos casos de prescrição com nome genérico, somente será permitida a dispensação do medicamento de referência ou de genérico correspondentes. É dever do profissional farmacêutico explicar detalhadamente a dispensação realizada ao paciente ou usuário, bem como fornecer toda a orientação necessária ao consumo racional do medicamento genérico. A substituição do genérico deverá pautar-se na relação de medicamentos genéricos registrados pela Anvisa. A relação de medicamentos genéricos deverá ser divulgada pela Anvisa por intermédio dos meios de comunicação.

\section{Benefício do uso de genéricos}

O uso de genéricos reduz substancialmente o custo das medicações para consumidores e sistemas de saúde.

Em 2000, os genéricos foram responsáveis por $42 \%$ de todas as prescrições nos EUA, mas somente por 85 dos 141 bilhões gastos na terapêutica medicamentosa. Estima-se que $\$ 1,16$ bilhão possa ser economizado para cada $1 \%$ de aumento no uso de drogas genéricas <www. ama-assn.org/ama/pub/category/15279.html>.4

Pode-se exemplificar a queda no preço e nos gastos após a introdução no mercado americano dos genéricos da gabapentina. $O$ gasto com medicação de marca da gabapentina em 2004 foi de $\$ 2.800$ milhões, sendo que em 2005, com a introdução do genérico, foi de $\$ 1.400$ milhões. Esta redução possibilita, ao menos potencialmente, um deslocamento de recursos para utilização em outras áreas na saúde.

Neste ambiente, a competição entre drogas genéricas e de marca leva a clara redução do custo das medicações de marca no mercado e também a um estímulo para a inovação e a descobertas de novos e melhores medicamentos.

\section{Preocupação com o uso de genéricos}

Há uma incerteza disseminada sobre a troca por medicamentos genéricos, com debate fervoroso sobre o assunto na literatura. ${ }^{5}$ Numa pesquisa com 6.420 neurologistas, dos 301 que responderam, $67 \%$ relataram recidiva das crises e $56 \%$ relataram aumento de efeitos adversos após a troca por medicamentos genéricos. ${ }^{6}$ Em outra pesquisa foram questionados 2.285 pacientes. Dos 1330 que responderam, 
$70 \%$ não relataram nenhum problema e $11 \%$ relataram aumento de crises ou de efeitos adversos após o início do uso de genéricos.?

Há relatos de preocupação pela substituição por genéricos. ${ }^{8,9,10}$ Relatos de casos e de séries indicam falha terapêutica depois da mudança para genéricos. ${ }^{11}$

Um artigo canadense sobre a mudança compulsória de DAE para genérico estudou a taxa de volta para o composto de marca, em comparação com outras classes de drogas. Estes dados foram comparados aos de antihiperlipidêmicos e antidepressivos. A taxa de volta em pacientes que mudaram para genéricos da lamotrigina e voltaram para o Lamictal foi de $12.9 \%$, e ao redor de $20 \%$ com valproato e clobazam, enquanto que foi 1,5 a 2,9\% a taxa de reconversão de antihiperlipidêmicos e antidepressivos. A média de aumento da dose de lamotrigina depois da substituição foi de $6,2 \%(\mathrm{p}<0.0001)$. Os autores comentam que há limitações metodológicas para serem retiradas conclusões definitivas. ${ }^{9}$

Entretanto, o FDA não encontrou nenhum caso documentado de falha terapêutica após substituição por genéricos ${ }^{11} \mathrm{e}$ o único ensaio clínico randomizado comparando DAE genérica e de marca (VPA) falhou em mostrar diferença. ${ }^{12}$

As preocupações reais são os limites da bioequivalência convencional $(-20 \mathrm{a}+25 \%$ do nível obtido com a mesma dose do medicamento de referência), que podem ser muito amplos para DAE, dadas as conseqüências da recorrência de crises. Esta faixa ampla indica que há riscos de mudança de genérico para genérico, pois eles podem estar situados nos extremos opostos da faixa de bioequivalência, ocasionando grandes variações nos níveis séricos de antiepilépticos.

Riscos de recorrência de crises e toxicidade das DAE levaram várias sociedades nacionais de epilepsia a formular recomendações para o uso de genéricos, nas quais preconizam cuidado contra a troca de formulações, especialmente em pacientes livres de crises. ${ }^{11}$

Há fatores adicionais na população de pacientes com epilepsia, tais como ansiedade e medo de ocorrência de novas crises, bem como perda da carteira de motorista, acidentes, injúrias e perda do emprego, caso ocorram recidivas das crises.

Genéricos comprovaram bioequivalência com o fármaco de referência, mas não entre si.

Há preocupação especial com produtos de liberação lenta, uma vez que estes produtos têm farmacocinética diferenciada dos demais. ${ }^{13}$

A qualidade dos genéricos não é uniforme mundialmente. Espera-se que maiores preocupações ocorram em paises em desenvolvimento, onde o controle de qualidade é aquém do desejado. Por outro lado, sua manutenção no mercado ao longo de anos, pode também ser menos provável do que a de formulações de referência.
A Academia Americana de Neurologia por vários dos motivos acima expostos, em recente parecer colocou-se contra a conduta da substituição de DAE por medicamentos genéricos sem a aprovação do médico atendente. ${ }^{14}$ Entretanto esta posição foi debatida por outros ${ }^{15}$ argumentando que não há fortes evidências para apoiar a posição da AAN e que estudos cegos, randomizados, e não pesquisas isoladas de opiniões, são necessários para esclarecer a questão concreta se genéricos de DAE levam a diferentes níveis séricos em comparação às drogas de referência. Estes mesmos autores discutem a influência financeira neste debate. Ao formular posições oficiais de nossas sociedades devemos evitar a percepção de conflitos atendo-nos às diretrizes proposta pelo FDA (Food and Drug Administration)..$^{16}$

Com relação aos pacientes com epilepsia recém-diagnosticada e virgens de tratamento medicamentoso acredita-se que possam ser tratados com medicação genérica, desde que os pacientes possam continuar com a mesma preparação. ${ }^{17}$

$\mathrm{O}$ assunto permanece aberto à discussão uma vez que faltam estudos metodologicamente adequados para responder as questões fundamentais sobre o tema. Apesar disto, e, levando em conta a experiência da literatura e a vivência pessoal dos membros da comissão, propomos as seguintes conclusões.

\section{CONCLUSÕES}

1. As formulações genéricas podem trazer enormes benefícios aos pacientes e à sociedade pela redução significativa dos custos dos medicamentos.

2. A prescrição de medicamentos genéricos é racional em pacientes com epilepsia recém-diagnosticada.

3. A substituição de medicamentos de marca, genéricos e similares pode ser justificada em pacientes que não estão com as crises epilépticas completamente controladas.

4. A substituição por medicamentos de marca, genéricos e similares deve ser evitada em pacientes livres de crises.

5. A mudança entre produtos de marca, genéricos e similares pode ser problemática.

6. Como os produtos de liberação lenta possuem uma farmacocinética mais complexa, deve-se evitar a sua troca.

7. Qualquer mudança de medicamentos deve ser avaliada pelo médico prescritor, considerando as peculiaridades da epilepsia e do paciente.

\section{REFERÊNCIAS}

1. <http://www.anvisa.gov.br/hotsite/genericos/index.htm $>$.

2. <http://e-legis.bvs.br/leisref/public/home.php $>$. 
3. <www.anvisa.gov.br/divulga/informes/2008/250308_2.htm>.

4. <www.ama-assn.org/ama/pub/category/15279.html>.

5. Bialer M. Generic products of antiepileptic drugs (AEDs): is it an issue? Epilepsia 2007;48:1825-1832.

6. Wilner NA. Therapeutic equivalency of generic antiepileptic drugs: results of a survey. Epilepsy Behav 2004;5(6):995-998.

7. Crawford P, Hall WW, Chappell B, Collings J, Stewart A. Generic prescribing for epilepsy. Is it safe? Seizure 1996;5(1):1-5.

8. Crawford P, Feely M, Guberman A, Kramer G. Are there potential problems with generic substitution of antiepileptic drug?: a review of issues. Seizure 2006; 15:165-176.

9. Andermann F, Duh MS, Gosselin A, Paradis PE. Compulsory generic switching of antiepileptic drugs: high switchback rates to branded compounds compared with other drug classes. Epilepsia 2007;48(3):464-469.

10. Berg MJ. What's the problem with the generic antiepileptic drugs? A call to action. Neurology 2007;68(16):1245-1246.

11. Perucca E, Albani F, Capovilla G, Dalla Bernardina B, Michelucci R, Zaccara G. Recommendations of the Italian League Against Epilepsy working group on generic products of antiepileptic drugs. Epilepsia. 2006;47(Suppl 5):S16-S20.

12. Vadney VJ, Kraushaar KW. Effects of switching from Depakene to generic valproic acid on individuals with mental retardation. Ment Retard 1997;35(6):468-472.
13. Mayer T, May TW, Altenmuller DM, Sandmann M, Wolf P. Clinical problems with generic antiepileptic drugs: comparison of sustainedrelease formulations of carbamazepine. Clin Drug Invest 1999;18: $17-26$.

14. Liow K, Barkley GL, Pollard JR, Harden CL, Bazil CW. Position statement on the coverage of anticonvulsant drugs for the treatment of epilepsy. Neurology 2007;68:1249-1250.

15. Miller JW, Anderson GD, Doherty MJ, Poolos NP. Correspondence. Position statement on the coverage of anticonvulsivant drugs for the treatment of epilepsy. What's the problem with generic antiepileptic drugs? A call to action. Neurology 2007;69: 1806-1808.

16. Harris G. FDA limits role of advisers tied to industry. The New York Times March 22, 2007.

17. Wolf P. Should newly diagnosed epilepsy be treated with generics? Nature Clinical Practice Neurology. Website: <www.nature.com./ clinicalpractice/neuro>. 26 fev. 2008.

\section{Endereco para correspondência:}

Carlos A. M. Guerreiro

Departamento de Neurologia da FCM-UNICAMP

Rua Tessália V. Camargo,126 - CP 611

CEP 13083-970, Campinas, SP, Brasil

guerreiro@fcm.unicamp.br 


\title{
JECN \\ Informações da Secretaria
}

\begin{abstract}
Prezado Sócio!
O Journal of Epilepsy and Clinical Neurophysiology (JECN) continua a campanha junto aos seus sócios solicitando a participação e a colaboração para enviarem artigos de revisão, artigos originais, relatos de casos, cartas e notícias sobre Epilepsia e áreas afins, que acharem necessária a divulgação.
\end{abstract}

PARTICIPE!

\section{Enviar para:}

Dr. Luciano De Paola (Editor)

Serviço de EEG - Hospital de Clínicas da UFPR

Rua Gen. Carneiro, 181

CEP 80060-900, Curitiba, PR, Brasil

E-mail: Ilpaola@mps.com.br

Poderá ser enviada também pelo e-mail:

jecnpoa@terra.com.br

(A/c. Secretária Sra. Nurma Ramos Pereira)

\section{JECN na sede própria}

Av. Montenegro, 186 sala 505 - Petrópolis

CEP 90460-160, Porto Alegre, RS, Brasil

Fone/Fax: (51) 3331-0161

E-mail: jecnpoa@terra.com.br

\section{HORÁRIO DE ATENDIMENTO:}

$2^{\mathrm{a}}$ a $5^{\mathrm{a}}$ feira: $14 \mathrm{~h}$ às $18 \mathrm{~h}$

$6^{a}$ feira: $14 \mathrm{~h}$ às $17 \mathrm{~h} 30 \mathrm{~min}$ 